\title{
Coronary vessels and cardiac myocytes of middle-aged rats demonstrate regional sex-specific adaptation in response to postmyocardial infarction remodeling
}

Eduard I Dedkov ${ }^{1 *}$, Kunal Oak', Lance P Christensen ${ }^{2}$ and Robert J Tomanek ${ }^{2}$

\begin{abstract}
Background: An increasing body of evidence indicates that left ventricular (LV) remodeling, especially the degree of reactive myocardial hypertrophy after myocardial infarction (MI), differs in males and females. Surprisingly, to date, the sex-specific post-MI alterations of the coronary vasculature remain undetermined. Therefore, we tested the hypothesis that adaptive coronary arteriolar and capillary modifications occurring in response to reactive myocyte hypertrophy differ between middle-aged male and female post-MI rats.
\end{abstract}

Methods: A large MI was induced in 12-month-old male (M-MI) and female (F-MI) Sprague-Dawley rats by ligation of the left coronary artery. Four weeks after surgery, rats with transmural infarctions, greater than $50 \%$ of the LV free wall (FW), were evaluated. Sham-operated male (M-Sham) and female (F-Sham) rats served as an age-matched controls.

Results: F-MI and M-MI rats had similar sized infarcts $(61.3 \% \pm 3.9 \%$ vs. $61.5 \% \pm 1.2 \%)$ and scale of LV remodeling, as indicated analogous remodeling indices (1.41 \pm 0.11 vs. $1.39 \pm 0.09)$. The degree of reactive post-Ml myocardial hypertrophy was adequate to normalize LV weight-to-body weight ratio in both sexes; however, the F-MI rats, in contrast to males, showed no myocyte enlargement in the LVFW epimyocardium. At the same time, a greater than $50 \%$ expansion of myocyte area in the male epimyocardium and in the female endomyocardium was accompanied by a 23\% $(P<0.05)$ increase in capillary-to-myocyte ratio, indicative of adaptive angiogenesis. Based on arteriolar length density in post-MI hearts, the resistance vessels grew in the male LVFW as well as the septum by $24 \%$ and 29\%, respectively. In contrast, in females, a significant (30\%) expansion of arteriolar bed was limited only to the LVFW. Moreover, in F-MI rats, the enlargement of the arteriolar bed occurred predominantly in the vessels with diameters $<30 \mu \mathrm{m}$, whereas in M-MI rats, a substantial (two- to threefold) increase in the density of larger arterioles (30 to $50 \mu \mathrm{m}$ in diameter) was also documented.

Conclusion: Our data reveal that while both sexes have a relatively similar pattern of global LV remodeling and adaptive angiogenesis in response to a large $\mathrm{Ml}$, male and female middle-aged rats differ markedly in the regional scale of reactive cardiac myocyte hypertrophy and adaptive arteriogenesis.

Keywords: Sex-related differences, Myocardial infarction, Left ventricular remodeling, Myocardial fibrosis, Cardiac myocyte hypertrophy, Coronary angiogenesis, Coronary arteriogenesis

\footnotetext{
* Correspondence: ededkov@nyit.edu

'Department of Biomedical Sciences, New York Institute of Technology College of Osteopathic Medicine, Rockefeller Building, Room 215E, Northern Boulevard, Old Westbury, NY 11568-8000, USA

Full list of author information is available at the end of the article
} waiver (http://creativecommons.org/publicdomain/zero/1.0/) applies to the data made available in this article, unless otherwise stated. 


\section{Background}

A large transmural myocardial infarction (MI) of the left ventricle (LV) initiates progressive structural remodeling, consisting of scarring and thinning of the infarcted region, dilatation of the LV chamber, reactive fibrosis, and compensatory hypertrophy in the noninfarcted LV myocardium [1-4]. It has also been established that the compensatory growth of cardiac myocytes in noninfarcted LV myocardium is often accompanied by the adaptive growth of coronary microvessels (capillaries and/or arterioles) $[5,6]$. The anatomical expansion of the vascular bed occurs primarily in the areas containing enlarged cardiac myocytes and compromised blood perfusion [7-9], indicating a strong functional link between the post-MI myocardial growth and the adaptive expansion of coronary vasculature [10].

In recent years, the accumulating body of knowledge derived from experimental animal studies and clinical research has revealed that the adaptive responses to post-MI remodeling differ between males and females [11]. For example, female rats and mice with a large transmural MI had less compensatory hypertrophy in noninfarcted LV regions than did males [12-14]. In concert with these experimental data, studies on humans have also demonstrated that women with LV dysfunction, caused in part by previous MI, were characterized by a smaller increase in LV mass compared to their respective male counterparts $[15,16]$. Surprisingly, despite the fact that males and females differ markedly in the extent of post-MI compensatory myocardial growth, there are no published studies investigating the degree of the adaptive coronary vessel growth between the sexes.

Furthermore, since MI is most common in middle-aged and senescent individuals of both genders $[17,18]$, the analysis of adaptive changes in post-MI hearts of middleaged, rather than young, animals is of greater relevance to the human population. Unfortunately, the majority of previously conducted MI experiments utilized either young or young adult animals. However, aged experimental animals, such as mice and rats, have been shown to differ markedly from their younger counterparts with regard to post-MI remodeling, including the level of LV hypertrophy $[19,20]$, the degree of reactive myocardial fibrosis [20], and adaptive coronary vessel growth $[6,21,22]$.

Accordingly, the aim of the current study was to test the hypothesis that sex is a determinant of adaptive responses of cardiac myocytes and coronary microvessels (capillaries and arterioles) in the noninfarcted LV myocardium after a large transmural MI in middle-aged rats.

\section{Methods}

All animal procedures were performed in accordance with the Guide for the Care and Use of Laboratory Animals published by the US National Institute of Health (NIH
Publications No. 85-23, revised 1996) and approved by the University of Iowa Animal Care and Use Committee.

\section{Animals and experimental model}

All experiments were conducted on 12-month-old (middle-aged) Sprague-Dawley rats of both sexes (Harlan, Indianapolis, IN, USA). Although in this study we did not assess plasma estradiol levels in our female animals, previous studies have determined that at this age, the rats are usually premenopausal but start to exhibit irregular estrous cycles $[23,24]$ similar to middle-aged women during the perimenopausal period [25].

Female (F-MI, $n=9)$ and male (M-MI, $n=9)$ rats were anesthetized with a mixture of ketamine $(100 \mathrm{mg} / \mathrm{kg}$ i.p.) and xylazine $(10 \mathrm{mg} / \mathrm{kg}$ i.p.) and the left coronary artery was permanently ligated near its origin, as previously detailed [26]. The mortality rate among post-MI rats was approximately $22 \%$ in males and approximately $11 \%$ in females, with all deaths occurring within the first $48 \mathrm{~h}$ after surgery. Sham-operated female (F-Sham, $n=6)$ and male (M-Sham, $n=7$ ) rats served as age-matched controls. Following surgery, the rats were housed under climatecontrolled conditions at a 12-h light/dark cycle and provided with standard rat chow and water ad libitum.

The rats were sacrificed 4 weeks after surgery, and the hearts were processed for comprehensive morphological analysis. All data from the noninfarcted LV free wall (FW) were derived from tissue approximately 1.5 to $2 \mathrm{~mm}$ distal from the infarct edge (Figure 1).

\section{Ventricular weight measurement, infarct size estimation, and tissue sampling}

The heart was arrested in diastole by the infusion of $2 \%$ lidocaine $\mathrm{HCl}$ into the left ventricle, then excised and perfuse-fixed on a Langendorff apparatus under constant pressure $(100 \mathrm{mmHg})$ for $20 \mathrm{~min}$ with $4 \%$ paraformaldehyde (PFA) in phosphate-buffered saline (PBS). The hearts were stored in a fresh $4 \%$ PFA solution for $24 \mathrm{~h}$ at $+4^{\circ} \mathrm{C}$. The atria were removed, and the right ventricular FW and the left ventricle (LVFW plus septum) were briefly blotted dry with filter paper and separately weighed. The left ventricle was then cut transversely into five parallel slices with a multiblade guillotine.

In hearts with MI, the all LV slices were digitized and infarct size was estimated using Image-Pro Analyzer 7.0 software (Media Cybernetics, L.P., Silver Spring, MD, USA), as detailed previously [26]. Briefly, in each digitized slice, the lengths of the entire free wall and its portion occupied by the scar (both obtained at the midwall level) were measured, and the extent of the scarred area was estimated as the ratio between scar length and length of the entire free wall. The mean of these ratios was calculated for each heart. Finally, the infarct size was expressed as a percentage of the LVFW. The data 


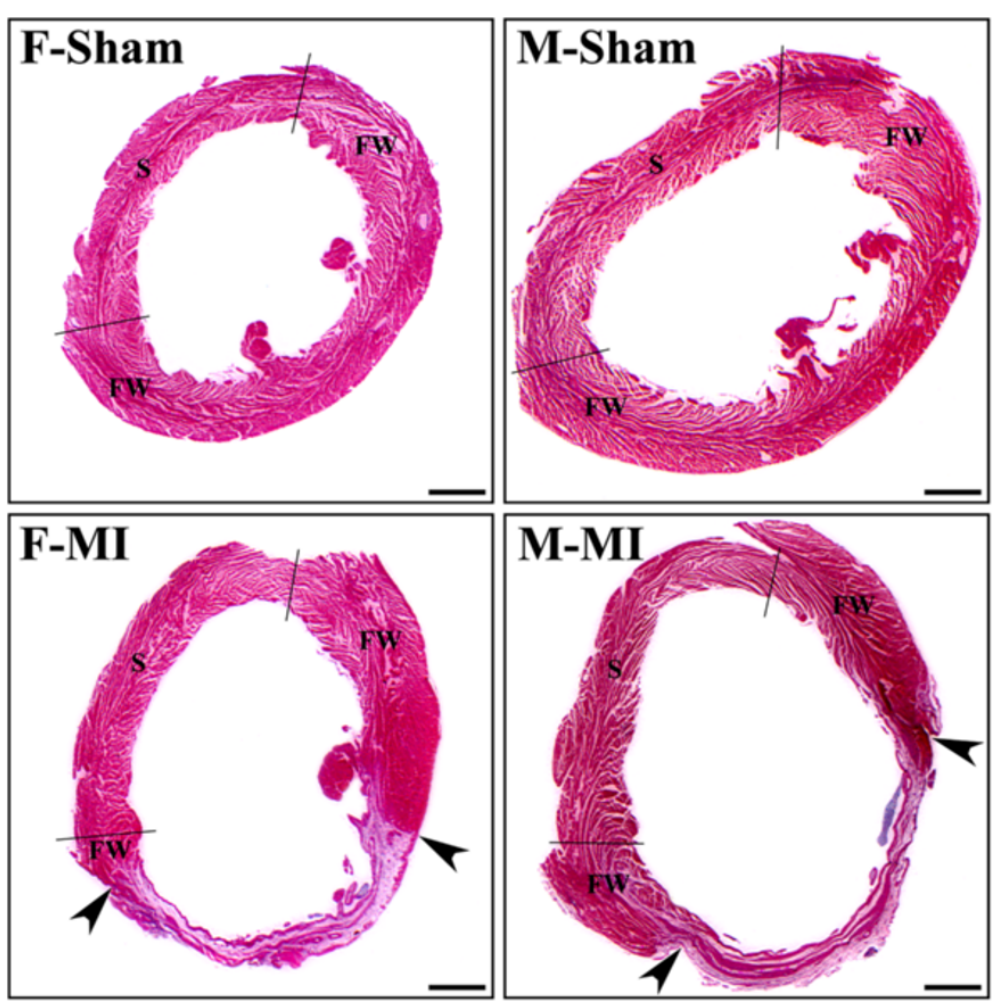

Figure 1 H\&E-stained cross sections of the left ventricle from sham and MI hearts of the rats. Female (F) and male (M). In each image, two black lines separate the interventricular septum (S) from the LV free wall (FW). In post-MI hearts, the arrowheads indicate the region of a transmural scar. Scale bars are $1.5 \mathrm{~mm}$.

from post-MI rats of both sexes were included in the study only if the infarct size was equal or greater than $50 \%$ of the LVFW. According to infarct size measurements, the seven hearts from M-MI rats and six hearts from F-MI rats were selected for final evaluation.

From each heart, two midventricular slices (at the level of the papillary muscles) were processed and embedded in paraffin, because they are most representative of the scale of LV remodeling and the extent of the transmural scar [27].

\section{Histology, immunohistochemistry, and microscopy}

Transverse $8.0-\mu \mathrm{m}$-thick serial sections were cut from paraffin-embedded LV slices and mounted onto microscope slides. Hematoxylin and eosin (H\&E) and Masson's trichrome stains were used for general morphological evaluation. Picrosirius red stain was utilized to visualize interstitial and periarteriolar collagen fibrils.

Additional sections were either immunostained with a Cy3-conjugated anti-smooth muscle (SM) $\alpha$-actin antibody (Sigma, St. Louis, MO, USA) to visualize the arterioles or double-labeled with a mixture of an Alexa Fluor 594conjugated Bandeiraea Simplicifolia-I (BSI) isolectin B4 (Molecular Probes, Inc., Eugene, OR, USA) and antilaminin antibody (Sigma, St. Louis, MO, USA) to visualize capillaries and to outline the cardiac myocytes, as detailed previously $[6,21,26]$. An Alexa Fluor 488-conjugated goat anti-rabbit antibody (Molecular Probes, Inc., Eugene, OR, USA) was used for visualization of a primary anti-laminin antibody. All sections were counterstained with DAPI (4', 6-Diamidino-2-Phenylindole; Vector Laboratories, Inc., Burlingame, CA, USA) to visualize the cell nuclei.

The stained sections were examined under the Olympus BX53 microscope (Shinjuku, Tokyo, Japan), and light and fluorescence images were captured into a computer using an Olympus DP72 digital camera (Shinjuku, Tokyo, Japan).

\section{Morphometric and stereological analyses}

Morphometric and stereological analyses were conducted on digitized images using Image-Pro Analyzer 7.0 software (Media Cybernetics, Inc., Bethesda, MD, USA).

The H\&E and Masson's trichrome-stained sections were used to obtain the following parameters of the left ventricle: (1) LV cross-sectional area (CSA) and diameter, (2) LV cavity CSA and mean cavity diameter, and (3) mean thickness of the LVFW and septum. From these measurements, we calculated the LV cavity diameter to septum thickness ratio [14] and the remodeling index detailed previously by Boyle and Weismann [28], i.e., (LV cavity CSA of MI heart/Average LV cavity CSA of Sham 
hearts $) \times($ LVFW thickness of MI heart/Average LVFW thickness of sham hearts).

The picrosirius red-stained sections were used to identify fibrillar collagen in interstitial and periarteriolar spaces. The interstitial collagen content was estimated as the volume fraction of the area occupied by fibrillar collagen and cardiac myocytes. The periarteriolar collagen content was defined as the ratio between the area occupied by the fibrillar collagen within the area surrounding the resistance vessel and the vessel area itself (media + lumen), as detailed previously [21,22]. For each heart, 14 to 20 resistance vessels (5 to $55 \mu \mathrm{m}$ in diameter) per myocardial region (i.e., LVFW and septum) were randomly selected and evaluated.

SM $\alpha$-actin-positive vessels (10 to $50 \mu \mathrm{m}$ in diameter) were used to calculate the arteriolar length, volume, and numerical densities, whereas vessels labeled with BSI-B4 isolectin (less than $5 \mu \mathrm{m}$ in minimal diameter) were used to estimate capillary numerical density, as previously detailed $[21,26]$. Arteriolar tortuosity (or an anisotropy coefficient) was then calculated as follows: arteriolar length density divided by arteriolar numerical density [29]. Cross-sectional area and numerical density of laminin-outlined transversely cut cardiac myocytes were determined in the same regions used for capillary analysis, and a capillary-to-myocyte ratio was calculated based on the numerical densities calculated for capillaries and cardiac myocytes, as previously detailed $[21,26]$. All parameters were estimated separately for the following three myocardial regions: LV epi- and endomyocardium and the septal endomyocardium. Only the areas in which the cardiac myocyte profiles demonstrated the relatively transverse plane and the presence of centrally located nuclei were used for evaluation. On average, approximately 280 to 310 myocyte profiles per region were counted in each heart.

\section{Statistical analysis}

Data are expressed as the mean \pm SEM. Statistical analysis was performed using IBM SPSS Statistics 22.0 software package (IBM Corp., Armonk NY, USA). Two-way analysis of variance (ANOVA) was performed to determine the interaction effect of sex (males vs. females) and the experimental model (Sham vs. MI) on the dependent variables. If a statistically significant difference was detected in the interaction effect and/or the main effects of sex and experimental model, a post hoc analysis was performed using Student's $t$ test with Bonferroni multiple comparison correction. $P<0.05$ was selected to denote significant differences.

\section{Results}

Considering the fact that middle-aged female and male rats had significantly different body weights, left and right ventricular weights as well as LV chamber dimensions (Table 1), our main emphasis was to conduct side-by-side comparison of sex-specific adaptations that occurred in the left ventricle of each sex group during cardiac remodeling triggered by a large transmural MI.

\section{LV structural remodeling and myocardial fibrosis}

Four weeks after left coronary artery occlusion, the mean infarct size was comparable between male and female rats (Table 1). The loss of cardiac myocytes was compensated by LV hypertrophy in both post-MI groups, as indicated unchanged LV weight-to-body weight ratios, that were similar to a corresponding group of sham rats. Furthermore, unaltered LV weight-to-ventricular weight ratios revealed that the left ventricles of both sexes had undergone a relatively proportional enlargement during 4 weeks of post-MI remodeling. It also appears that the compensatory LV growth in male and female rats was primarily longitudinal, since the transverse LV chamber dimensions and thickness of the noninfarcted myocardial wall remained relatively similar to the sham values. Most importantly, even though male post-MI rats, in contrast to female animals, showed a significant expansion of the LV cavity (Figure 1), a global pattern of LV chamber remodeling remained comparable between two sexes, as indicated by nearly identical values for remodeling index and LV cavity diameter to septum thickness ratio (Table 1).

Post-MI remodeling in the noninfarcted LV myocardium of both sexes was associated with an increase in the content of interstitial fibrillar collagen (Figure 2A,B). As shown in Figure $2 \mathrm{C}$, the fractional volume of interstitial collagen was higher in post-MI rats by $13 \%$ in male and $37 \%$ in female free wall and $23 \%$ and $34 \%$ in male and female septum, respectively, as compared with corresponding sham rats (Figure 2C). In contrast, periarteriolar collagen revealed a marked (2.5- to threefold) accumulation only in post-MI female rats (Figure 2D). In spite of a more exaggerated accumulation of interstitial and periarteriolar collagen in surviving myocardium, the post-MI female rats had significantly smaller fractional volume of fibrillar collagen in both the LV free wall and septum compared to their age-matched male counterparts. This was in part due to the fact that the fractional volume of interstitial and periarteriolar collagen was much lower in female rats prior to infarction, as indicated by the values in shams (Figure 2C,D).

\section{Capillary bed adaptation in response to regional cardiac myocyte hypertrophy}

Despite the fact that in both sexes, average thickness of the noninfarcted LV wall remained comparable to agematched sham rats (Table 1), cardiac myocytes in the noninfarcted free wall myocardium of the male rats were significantly enlarged in both the epimyocardium (57\%) 
Table $1 \mathrm{Global}$ parameters of the left ventricle in shame and MI hearts from male $(\mathrm{M})$ and female (F) rats

\begin{tabular}{|c|c|c|c|c|}
\hline & M-Sham & M-MI & F-Sham & F-MI \\
\hline Number & 7 & 7 & 6 & 6 \\
\hline Infarct size, \% of LVFW & - & $61.5 \pm 1.2(57.5-63.8)$ & - & $61.3 \pm 3.9(50.0-71.3)$ \\
\hline$B W, g$ & $560.0 \pm 15.4$ & $536.8 \pm 27.2$ & $362.5 \pm 6.4^{\S \S \S}$ & $335.7 \pm 21.5^{++\dagger}$ \\
\hline $\mathrm{W}, \mathrm{mg}$ & $1476.4 \pm 39.7$ & $1461.8 \pm 88.2$ & $1214.5 \pm 50.6^{\S}$ & $1084.2 \pm 16.6^{t+\dagger}$ \\
\hline LWW, mg & $1150.4 \pm 33.4$ & $1100.4 \pm 55.2$ & $935.2 \pm 46.0^{\S \S}$ & $829.5 \pm 12.2^{\dagger+\dagger}$ \\
\hline RWW, mg & $326.0 \pm 9.6$ & $361.4 \pm 40.5$ & $279.3 \pm 5.6$ & $254.7 \pm 18.3^{\dagger}$ \\
\hline LWW/BW, mg/g & $2.06 \pm 0.08$ & $2.05 \pm 0.07$ & $2.58 \pm 0.09^{\S}$ & $2.52 \pm 0.15^{\dagger}$ \\
\hline LWW/W, mg/mg & $0.78 \pm 0.01$ & $0.76 \pm 0.02$ & $0.77 \pm 0.01$ & $0.77 \pm 0.01$ \\
\hline LV diameter, $\mathrm{mm}$ & $11.06 \pm 0.09$ & $11.03 \pm 0.25$ & $9.89 \pm 0.24^{\S}$ & $9.88 \pm 0.19^{\dagger \dagger}$ \\
\hline LV CSA, mm² & $96.9 \pm 1.6$ & $96.5 \pm 4.3$ & $78.3 \pm 4.0^{\S}$ & $78.4 \pm 3.1^{\dagger}$ \\
\hline LV cavity diameter, mm & $6.33 \pm 0.30$ & $7.58 \pm 0.22^{*}$ & $5.82 \pm 0.34$ & $6.61 \pm 0.44^{\dagger}$ \\
\hline LV cavity CSA, mm ${ }^{2}$ & $32.9 \pm 2.9$ & $46.4 \pm 2.7^{*}$ & $27.9 \pm 3.1$ & $36.4 \pm 4.5^{\dagger}$ \\
\hline LVFW thickness, mm & $2.31 \pm 0.13$ & $2.29 \pm 0.11$ & $1.92 \pm 0.10$ & $2.12 \pm 0.21$ \\
\hline Septum thickness, mm & $1.75 \pm 0.09$ & $1.77 \pm 0.12$ & $1.64 \pm 0.08$ & $1.68 \pm 0.11$ \\
\hline LV cavity diameter/septum & $3.67 \pm 0.30$ & $4.39 \pm 0.35$ & $3.60 \pm 0.30$ & $4.02 \pm 0.44$ \\
\hline Remodeling index & - & $1.39 \pm 0.09$ & - & $1.41 \pm 0.11$ \\
\hline
\end{tabular}

Values are the mean \pm SEM; $n$, Number of rats; LVFW, left ventricular free wall; BW, body weight; VW, ventricular weight; LVW, left ventricular weight; RVW, right ventricular weight; CSA, cross-sectional area. Remodeling index = (LV cavity CSA of MI heart/Average LV cavity CSA of sham hearts) $\times$ (LVFW thickness of MI heart/ Average LVFW thickness of sham hearts). ${ }^{*} P<0.05$ vs. corresponding Sham group; ${ }^{\S} P<0.05,{ }^{\S \S} P<0.01$, and ${ }^{\S \S} P<0.001$ vs. M-Sham; ${ }^{\dagger} P<0.05$, ${ }^{\dagger \dagger} P<0.01$, and ${ }^{++\dagger} P<0.001$ vs. M-MI

and endomyocardium (38\%) compared to shams, whereas in post-MI female rats, a marked (62\%) compensatory growth of cardiac myocytes occurred only in the free wall endomyocardium (Figure 3A,B,C). While these data suggest that during the four post-MI weeks, both male and female rats experienced a substantial reduction in the number of cardiac myocytes across the LV wall, the loss of cardiac myocytes was less prominent in post-MI female rats, primarily in the free wall epimyocardium, compared to that in post-MI males (Table 2 and Figure 3A,B). At the same time, neither of the two post-MI groups revealed any concentric enlargement of cardiac myocytes in the septum (Table 2).

Generally, the transverse enlargement of cardiac myocytes in the LV free wall of post-MI male and female rats was associated with the reduction in capillary density (Table 2). Nevertheless, in both sexes, a greater than 50\% expansion in myocyte cross-sectional area was accompanied by a significant compensatory angiogenic response. As shown in Figure 3D, the capillary-to-myocyte ratio was $23 \%$ greater in both the male epimyocardium and the female endomyocardium, as compared to corresponding sham rats. At the same time, the unchanged values of capillary-to-myocyte ratio detected in other areas of the remodeled LV myocardium of both sexes (Table 2) suggested an adequate functional match between the cardiac myocyte size and the capillary density in these regions.

\section{Regional differences in arteriolar bed adaptation}

Post-MI remodeling of noninfarcted LV myocardium was associated with a substantial expansion of the coronary arteriolar bed in both sexes as indicated by a significant increase in arteriolar length density compared to corresponding sham values (Table 3). However, while in male rats, arteriolar density increased in both the LV free wall and the septum by $24 \%$ and $29 \%$, respectively, in females, a significant (30\%) expansion of the arteriolar bed occurred only in the LV free wall (Figure 4A,B,C). Furthermore, whereas in female rats arteriolar growth occurred predominantly in the vessels with diameters $<30 \mu \mathrm{m}$, in male rats, a substantial (two to threefold) increase occurred in larger arterioles (30 to $50 \mu \mathrm{m}$ in diameter) as well (Figure 4D).

It is important to emphasize the fact that although the arteriolar beds of the female sham and post-MI rats were markedly smaller than in the hearts of their age-matched male counterparts, we found a relatively similar pattern of arteriolar network organization in two sexes, as indicated by a comparable degree of arteriolar tortuosity (Table 3 ) and the nearly analogous scale of arteriolar frequency distribution in relation to a particular vessel diameter group (Figure 5). However, in contrast to male rats, postMI females showed a more significant shift in hierarchic distribution of arterioles within the vascular bed, especially in the LV free wall, as compared to sham rats. Such sex-specific arteriolar tree modification was further 

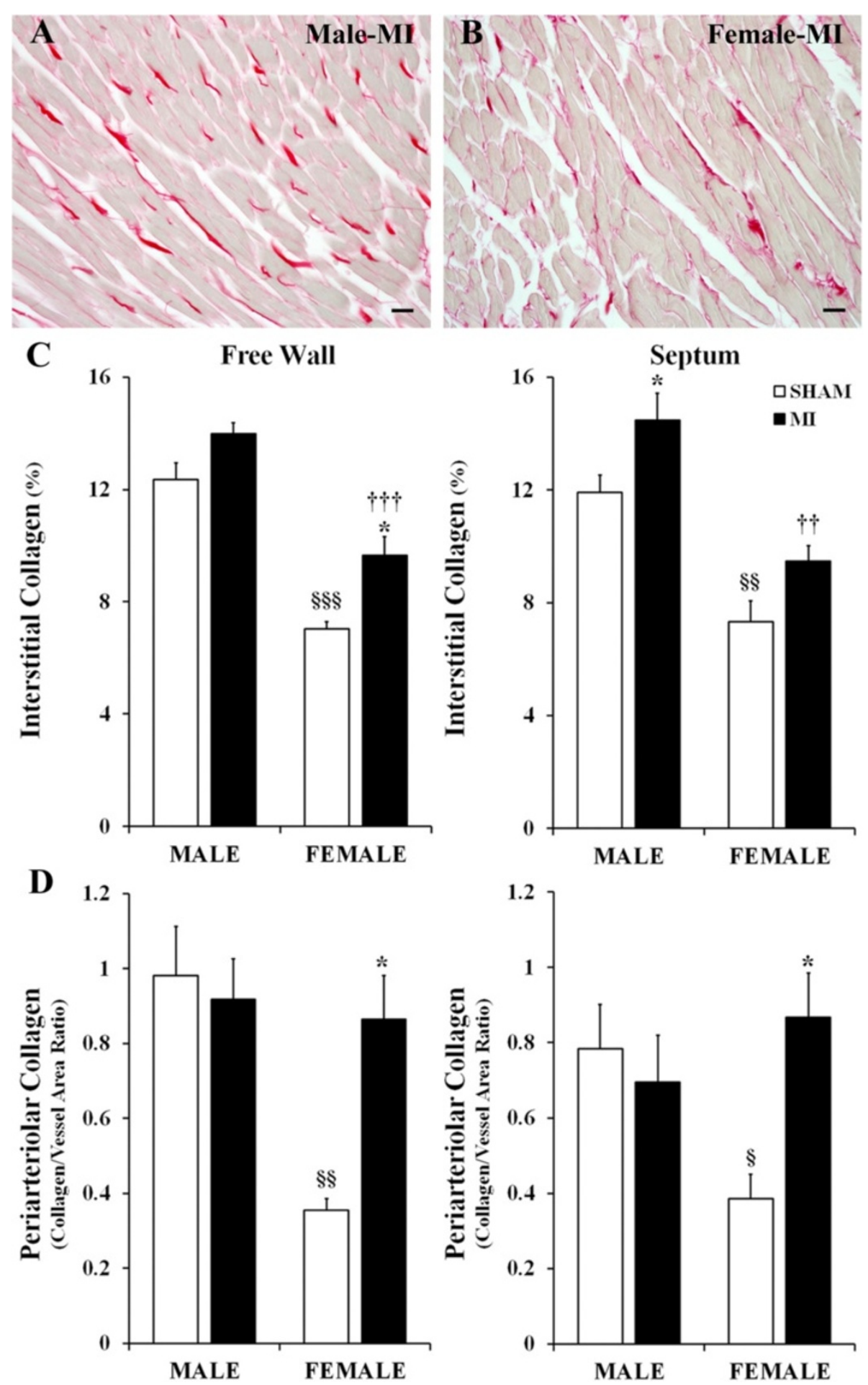

Figure 2 Extent of interstitial and periarteriolar fibrosis. (A,B) Representative images of picrosirius red-stained collagen fibers (bright red color) in the LV free wall myocardium of post-MI male and female rats. (C) Fractional volume of interstitial collagen fibers in the LV free wall and septum. (D) The content of periarteriolar collagen in the LV free wall and septum. Scale bars are $20 \mu \mathrm{m}$. Values are the mean \pm SEM; $n=7$ male rats/group; $n=6$ female rats/group. A two-way ANOVA revealed a statistically significant interaction between the effects of sex and the experimental model on perivascular collagen content in both $\mathrm{LV}$ free wall, $F(1,22)=6.860, P=0.020$, and the septum, $F(1,22)=5.876, P=0.028$. ${ }^{*} P<0.05$ vs. a corresponding sham group; ${ }^{\S} P<0.05,{ }^{\S \S} P<0.01$, and ${ }^{\S \S \S} P<0.001$ vs. M-Sham; ${ }^{\dagger+} P<0.01$ and ${ }^{+\dagger \dagger} P<0.001$ vs. M-Ml rats. 


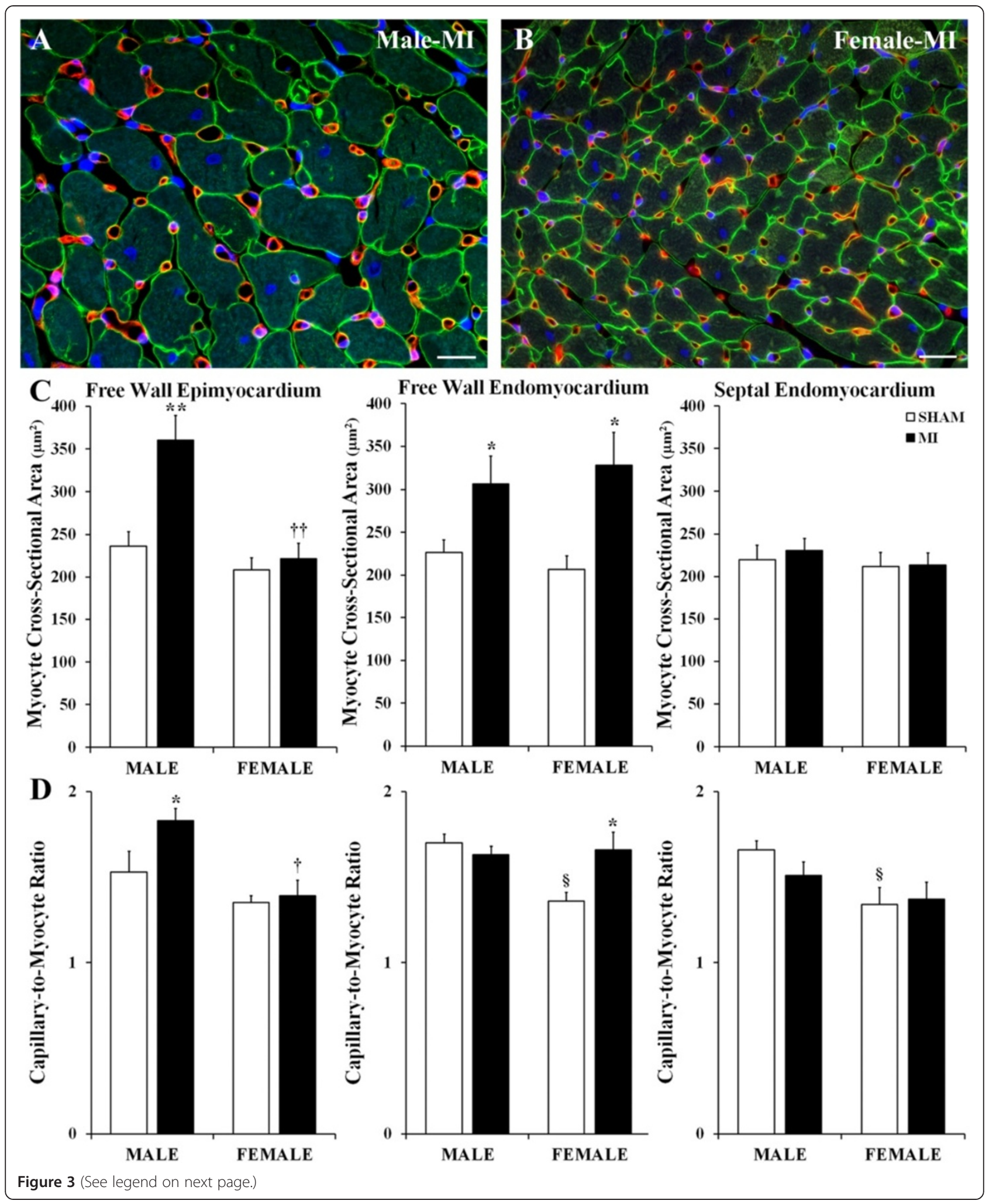


(See figure on previous page.)

Figure 3 Degree of reactive cardiac myocyte hypertrophy and compensatory angiogenesis. $(A, B)$ Representative images of the free wall epimyocardium of post-MI male and female hearts immunofluorescence-stained with an antibody against laminin (green color), BSI isolectin B4 (red color), and DAPI (blue). (C) Cross-sectional area of cardiac myocytes in the LV free wall and septum. (D) Capillary-to-myocyte ratios in the LV free wall and septum. Scale bars are $20 \mu \mathrm{m}$. Values are the mean \pm SEM; $n=7$ male rats/group; $n=6$ female rats/group. Note, in septal myocardium, all measurements were done on the endomyocardial side that is faced toward the LV cavity. A two-way ANOVA revealed a statistically significant interaction between the effects of sex and the experimental model on myocyte CSA in epimyocardial region, $F(1,22)=6.032, P=0.026$, and on the capillary-to-cardiac myocyte ratio in free wall endomyocardium, $F(1,22)=6.307, P=0.025 .{ }^{*} P<0.05$ and ${ }^{* *} P<0.01$ vs. a corresponding sham group; ${ }^{\S} P<0.05$ vs. M-Sham; ${ }^{\dagger} P<0.05$ vs. M-Ml rats.

exposed by the opposing patterns of changes within the large and small diameter vessel groups in two sexes (Figure 5).

\section{Discussion}

Data from this study provide four major findings. First, male and female middle-aged rats with a transmural MI of the comparably large size undergo a relatively similar pattern of global LV chamber remodeling and reactive myocardial hypertrophy; however, the myocardial content of fibrillar collagen, especially in periarteriolar regions, increases more in female than in male rats. Second, the transverse growth of cardiac myocytes in the LV free wall is limited to the endomyocardium of female post-MI rats, whereas male rats experience a marked enlargement of cardiac myocyte in both epimyocardial and endomyocardial regions. Third, post-MI rats of both sexes showed that significant adaptive angiogenesis occurs only in the areas of the LV free wall associated with the cross-sectional expansion of cardiac myocyte greater than $50 \%$. Finally, a significant expansion of the coronary arteriolar tree occurs in both the LV free wall and the septum of post-MI male rats, whereas in females, adaptive arteriogenesis is limited only to the free wall. Taken together, these findings support our conclusion that significant regional sex-related differences characterize the post-MI adaptive responses of various structural components in surviving LV myocardium, especially cardiac myocytes and coronary microvessels.

Does sex/gender affect the pattern of global post-MI LV remodeling and a regional hypertrophic response of the cardiac myocytes?

Although the structural alterations in LV architecture, termed ventricular remodeling, which occur in response to a large transmural MI, have been studied for the last three decades in both humans and experimental animals $[1-3,30]$, the findings related to sex/gender-specific differences during this process remain obscure $[11,31,32]$. Therefore, our study is the first to conduct a side-by-side comparison of the global and regional patterns of LV remodeling between post-MI male and female middleaged rats. We focused specifically on middle-aged animals because this age group, along with older individuals, corresponds to a human population, including both sexes, which are most vulnerable for myocardial ischemia and infarction $[17,18,33,34]$. We also chose to evaluate the hearts at the end of the first post-MI month because it

Table 2 Capillary and cardiac myocyte numerical densities and capillary-to-myocyte ratio in the left ventricle of shame and $\mathrm{MI}$ hearts from male $(\mathrm{M})$ and female $(\mathrm{F})$ rats

\begin{tabular}{|c|c|c|c|c|c|c|c|}
\hline & \multirow[t]{2}{*}{ Number } & \multicolumn{3}{|c|}{ LV free wall } & \multicolumn{3}{|c|}{ Septum } \\
\hline & & $\mathrm{CD}$, number $/ \mathrm{mm}^{2}$ & $\mathrm{MD}$, number $/ \mathrm{mm}^{2}$ & $\mathrm{C} / \mathrm{M}$ & $\mathrm{CD}$, number $/ \mathrm{mm}^{2}$ & $\mathrm{MD}$, number $/ \mathrm{mm}^{2}$ & $\mathrm{C} / \mathrm{M}$ \\
\hline \multicolumn{8}{|c|}{ Epimyocardium } \\
\hline M-Sham & 7 & $4,929.7 \pm 407.7$ & $3,142.9 \pm 193.6$ & $1.59 \pm 0.12$ & N/A & N/A & N/A \\
\hline $\mathrm{M}-\mathrm{Ml}$ & 7 & $3,818.1 \pm 169.9^{*}$ & $2,178.9 \pm 207.6^{*}$ & $1.81 \pm 0.08^{*}$ & N/A & N/A & N/A \\
\hline F-Sham & 6 & $5,405.5 \pm 418.9$ & $4,027.2 \pm 262.2^{\S}$ & $1.35 \pm 0.04$ & N/A & N/A & N/A \\
\hline F-Ml & 6 & $5,142.7 \pm 773.8$ & $3,661.4 \pm 312.3^{t+}$ & $1.39 \pm 0.09^{\dagger}$ & N/A & N/A & N/A \\
\hline \multicolumn{8}{|c|}{ Endomyocardium } \\
\hline M-Sham & 7 & $6,220.4 \pm 329.9$ & $3,739.6 \pm 195.2$ & $1.67 \pm 0.05$ & $6,107.4 \pm 459.6$ & $3,783.9 \pm 286.5$ & $1.62 \pm 0.04$ \\
\hline $\mathrm{M}-\mathrm{Ml}$ & 7 & $4,353.3 \pm 687.1^{*}$ & $2,704.7 \pm 351.0^{*}$ & $1.60 \pm 0.06$ & $5,215.2 \pm 592.3$ & $3,443.6 \pm 262.4$ & $1.52 \pm 0.09$ \\
\hline F-Sham & 6 & $5,477.4 \pm 396.5$ & $4,032.1 \pm 305.2$ & $1.36 \pm 0.05^{\S}$ & $5,361.5 \pm 908.9$ & $3,938.7 \pm 346.8$ & $1.34 \pm 0.12^{\S}$ \\
\hline F-MI & 6 & $4,492.8 \pm 648.0$ & $2,709.2 \pm 337.3^{*}$ & $1.66 \pm 0.13^{*}$ & $5,510.2 \pm 717.4$ & $3,875.4 \pm 359.8$ & $1.37 \pm 0.11$ \\
\hline
\end{tabular}

Values are the mean \pm SEM. $n$, number of rats; CD, capillary density; MD, myocyte density; C/M, capillary-to-myocyte ratio; N/A, mot applicable. Note that in septum all parameters were analyzed only in LV endomyocardium. A two-way ANOVA revealed a statistically significant interaction between the effects of sex and the experimental model on the capillary-to-cardiac myocyte ratio in free wall endomyocardium, $F(1,22)=6.307, P=0.025$. ${ }^{*} P<0.05$ vs. corresponding Sham group: ${ }^{5} P<0.05$ vs. M-Sham; ${ }^{\dagger} P<0.05$ and ${ }^{\dagger+} P<0.01$ vs. M-Ml. 
Table 3 Arteriolar diameter, numerical density, length density, volume densities, and the degree of arteriolar tortuosity in the left ventricle of shame and MI hearts from male (M) and female (F) rats

\begin{tabular}{|c|c|c|c|c|c|c|}
\hline & Number & Diameter minimum, $\mu \mathrm{m}$ & $\begin{array}{c}N_{\mathrm{A}^{\prime}} \\
\text { counts } / \mathrm{mm}^{2}\end{array}$ & $L_{\mathrm{V}}, \mathrm{mm} / \mathrm{mm}^{3}$ & $\begin{array}{c}\text { Tortuosity, } \\
L_{\mathrm{V}} / N_{\mathrm{A}}\end{array}$ & $V_{V}$, percent \\
\hline \multicolumn{7}{|c|}{ LV free wall } \\
\hline M-Sham & 7 & $15.7 \pm 0.6$ & $20.4 \pm 1.3$ & $54.6 \pm 2.3$ & $2.7 \pm 0.1$ & $1.22 \pm 0.16$ \\
\hline M-Ml & 7 & $16.4 \pm 0.4$ & $22.2 \pm 1.4$ & $66.8 \pm 4.8^{*}$ & $3.0 \pm 0.1$ & $1.55 \pm 0.15$ \\
\hline F-Sham & 6 & $19.5 \pm 0.7^{\S \S \S}$ & $6.9 \pm 0.3^{\S \S \S}$ & $18.6 \pm 1.6^{\S \S \S}$ & $2.7 \pm 0.2$ & $0.62 \pm 0.03^{\S}$ \\
\hline F-Ml & 6 & $17.0 \pm 0.4^{*}$ & $9.3 \pm 0.8^{t+\dagger}$ & $23.7 \pm 2.2^{*++\dagger}$ & $2.6 \pm 0.2$ & $0.63 \pm 0.06^{\dagger \dagger}$ \\
\hline \multicolumn{7}{|l|}{ Septum } \\
\hline M-Sham & 7 & $15.7 \pm 0.3$ & $17.5 \pm 1.4$ & $41.9 \pm 1.6$ & $2.4 \pm 0.1$ & $0.96 \pm 0.05$ \\
\hline M-MI & 7 & $16.2 \pm 0.3$ & $21.2 \pm 1.9$ & $53.6 \pm 5.7^{*}$ & $2.5 \pm 0.1$ & $1.38 \pm 0.11^{*}$ \\
\hline F-Sham & 6 & $17.9 \pm 0.5^{\S \S}$ & $11.0 \pm 1.1^{\S}$ & $25.8 \pm 1.4^{\S \S}$ & $2.4 \pm 0.2$ & $0.84 \pm 0.09$ \\
\hline F-Ml & 6 & $16.8 \pm 0.6$ & $8.9 \pm 0.9^{t+\dagger}$ & $23.0 \pm 2.2^{\dagger+\dagger}$ & $2.6 \pm 0.1$ & $0.61 \pm 0.12^{++\dagger}$ \\
\hline
\end{tabular}

Values are the mean \pm SEM. $n$, number of rats; $N_{\mathrm{A}}$, numerical density; $L_{\mathrm{V}}$, length density; $V_{\mathrm{V}}$, volume density. A two-way ANOVA revealed a statistically significant interaction between the effects of sex and the experimental model on the arteriolar diameter in $\operatorname{LV}$ free wall, $F(1,22)=8.943, P=0.009$, and on arteriolar volume density in septum, $F(1,22)=10.903, P=0.005$. ${ }^{*} P<0.05$ vs. corresponding Sham group; ${ }^{5} P<0.05,{ }^{{ }^{5}} P<0.01$, and ${ }^{\$ 55} P<0.001$ vs. M-Sham; ${ }^{\dagger} P<0.05,{ }^{\dagger \dagger} P<0.01$, and ${ }^{++\dagger} P<0.001$ vs. M-MI.

usually represents a compensated phase of the infarctinduced LV remodeling process. At this stage, the left ventricle contains a formed scar [32], hypertrophied cardiac myocytes, and significantly expanded cavity [2], but yet preserves the thickness of the noninfarcted myocardial wall [35] and the external cross-sectional chamber dimensions [36]. In agreement with these observations, we found that although the LV cavity of post-MI male and female middle-aged rats was expanded, the LV weight-to-body weight ratio, the external cross-sectional dimensions of the LV chamber, and the thickness of both the septal and noninfarcted free wall myocardium remained similar to shame values, suggesting a compensated stage of ventricular remodeling. Most importantly, we established the fact that despite a significant difference in the size of the left ventricles, the post-MI middle-aged male and female rats had virtually identical values of the remodeling indices and the LV cavity diameter to septum thickness ratio, indicative of a similar pattern of global LV remodeling between the sexes.

However, our data are in contrast to previous observations by others who studied young adult post-MI rats $[12,14,31]$. These studies noted a significant difference in the LV remodeling pattern between male and female animals. One study found a greater increase in the thickness of noninfarcted free wall in males compared to females [12], whereas another reported that males had a smaller cross-sectional expansion of the LV chamber than females [31]. We believe that discrepancy between our findings and those reported previously are attributable to the significant difference in the age of rats studied. This argument is supported by findings showing that LV parameters such as cavity dimensions, the size of cardiac myocytes, and the scale of myocardial fibrosis differ significantly between young and aged rodents (mice and rats) both at the onset of MI and during LV remodeling $[19,20]$. These data suggest that the high level of myocardial fibrosis in the hearts of sham-operated middle-aged rats, particularly males, is probably age-related since data from younger animals did not reveal extensive fibrosis [13]. Yet, our data are consistent with the fact that LV myocardium of the female rats, even after a large MI, revealed a lesser amount of interstitial collagen than males [13]. To a certain extent, this phenomenon can be attributed to an antifibrotic effect of estrogen [37], the level of which remains fairly high in middle-aged rats [23]. However, we found a significant interaction between the effects of sex and the experimental model on perivascular collagen content in both LV free wall and the septum. Particularly, we determined that post-MI remodeling in middle-aged female rats causes a much greater accumulation of interstitial and especially perivascular collagen than in males.

Since the compensatory recovery of LV weight in postMI male and female middle-aged rats occurred without noticeable thickening of the myocardial wall, it is likely that in both sexes, most of the compensatory myocardial growth occurred via cardiac myocyte lengthening, similar to that reported previously by Chen et al. [31] in young adult rats. However, in contrast to the latter study, we detected the marked regional differences in transverse myocyte areas, primarily in the noninfarcted free wall myocardium. One potential explanation for such discrepancy might be the fact that Chen and colleagues [31] analyzed cardiac myocytes isolated from both the free wall and the septum together, while in our study, we measured the myocyte cross-sectional areas separately in three LV regions (free wall epi- and endomyocardium; 

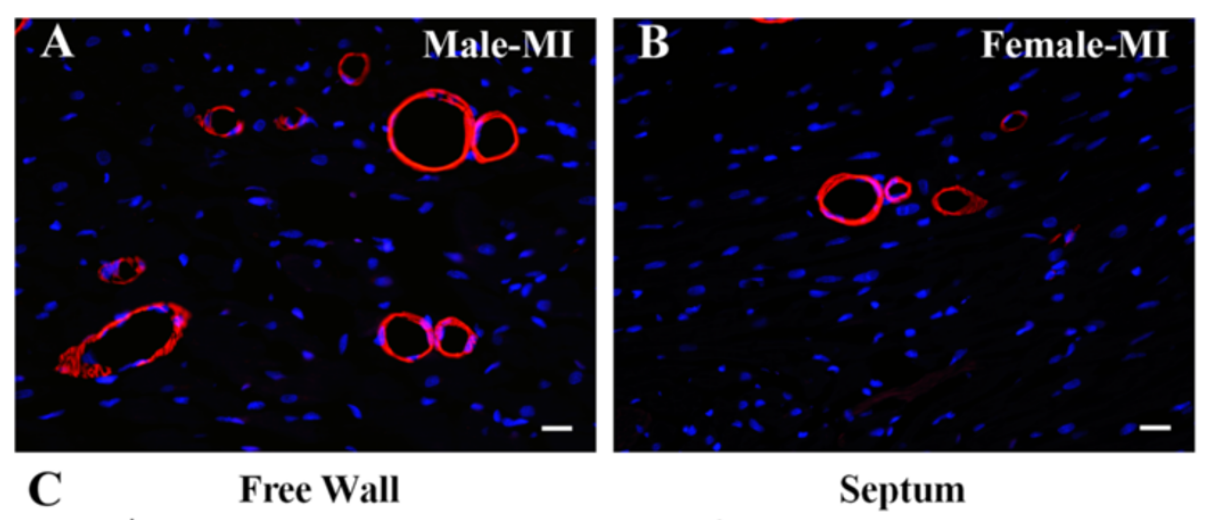

Septum
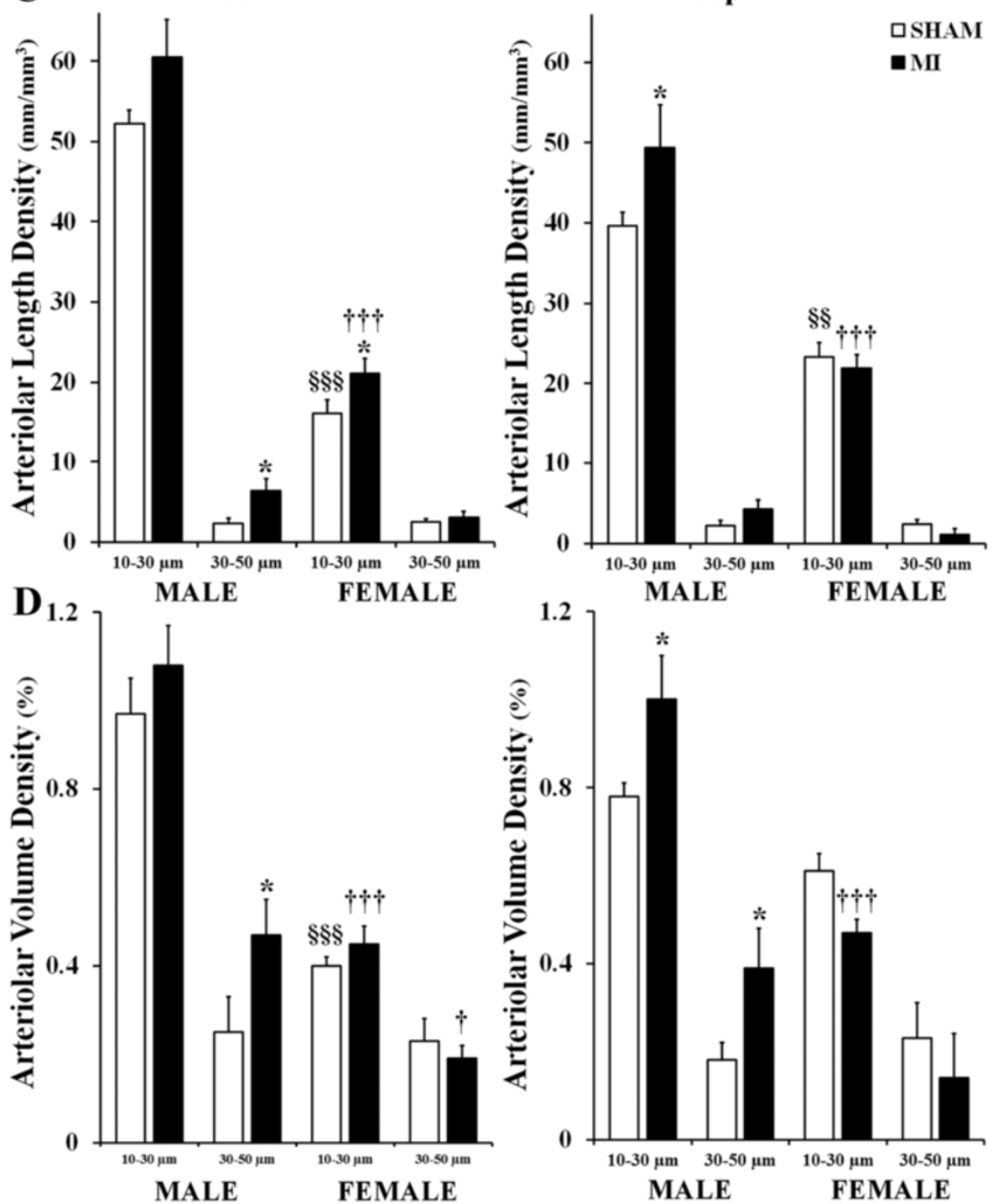

Figure 4 Scale of adaptive arteriogenesis. $(\mathbf{A}, \mathbf{B})$ Representative images of the LV free wall myocardium of post-MI male and female rats immunofluorescence-stained with an antibody against smooth muscle a-actin (red color) and DAPI (blue). (C) Arteriolar length density relative to a vessel diameter group in the LV free wall and septum. (D) Arteriolar volume density relative to a vessel diameter group in the LV free wall and septum. Scale bars are $20 \mu \mathrm{m}$. Values are the mean \pm SEM; $n=7$ male rats/group; $n=6$ female rats/group. ${ }^{*} P<0.05$ vs. a corresponding sham group; ${ }^{\S \S} P<0.01$ and ${ }^{\S \S \S} P<0.001$ vs. M-Sham rats; ${ }^{\dagger} P<0.05$ and ${ }^{+\dagger+} P<0.001$ vs. M-Ml rats. 


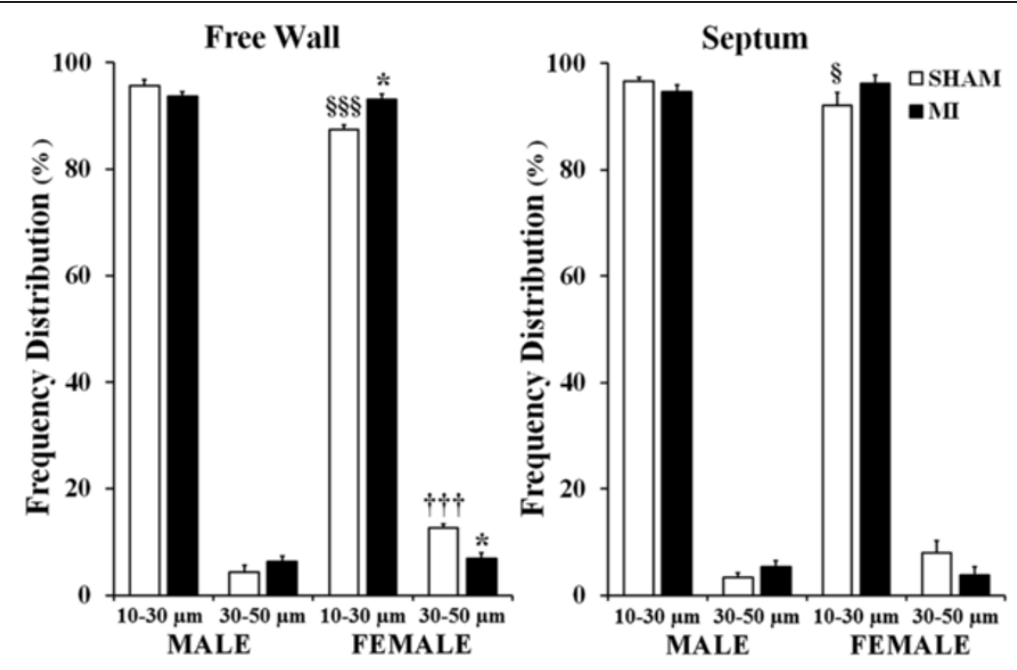

Figure 5 Frequency distribution of arterioles relative to a vessel diameter group. Values are the mean $\pm S E M ; n=7$ male rats/group; $n=6$ female rats/group. A two-way ANOVA revealed a statistically significant interaction between the effects of sex and the experimental model on the frequency distribution of arterioles in LV free wall, $F(1,22)=12.001, P=0.003 .{ }^{*} P<0.05$ vs. a corresponding sham group; ${ }^{\S} P<0.05$ and ${ }^{\S \S \S} P<0.001$ vs. M-Sham rats; ${ }^{\text {t+† }} P<0.001$ vs. M-Ml rats.

and septal endomyocardium). Besides, our data documenting post-MI compensatory growth of cardiac myocytes in the LV free wall are consistent with numerous previous studies $[5,12,13,38,39]$. However, in addition to these findings, our data revealed a significant interaction between the effects of sex and the experimental model on myocyte cross-sectional area in the epimyocardial region. Specifically, we established the fact that post-MI female rats, in contrast to males, had no reactive enlargement of cardiac myocytes in the epimyocardial region. Such distinction in myocyte hypertrophic response suggests the existence of a sex-specific difference in regional epicardial wall stress $[40,41]$ between the sexes. Taking into consideration the fact that regionally elevated wall stress can cause pronounced focal degradation of the extracellular matrix [40], it feasible to speculate that such areas of post-MI male hearts may be more prone to side-by-side slippage of myocyte bundles [42,43], increased apoptotic myocyte death [44] and, hence, exaggerated concentric hypertrophy of surviving cardiac myocytes [45].

\section{Does sex/gender influence a scale of coronary microvessels (capillary and arterioles) adaptation during post-MI remodeling?}

Over the past several decades, a large body of accumulating evidence has established the fact that the coronary vasculature undergoes structural adaptations, including angiogenesis and arteriogenesis, in response to postinfarction LV remodeling [46]. However, most of the published experimental studies used either male [39,47-49] or, rarely, female [50] animals only. Most surprising is the fact that, with the exception of our recent studies on middleaged rats $[6,21,22,26]$, the majority of earlier observations involving murine myocardium have been done on young or young adult mice or rats, which are known to have a more elaborated and highly adaptable coronary vascular network than the hearts of older animals [51-53]. Therefore, our current study is the first to address a sex-specific pattern of coronary microvessel adaptations in the left ventricle of post-MI middle-aged rats.

Consistent with the previous reports on younger animals $[39,47,49,54]$, we found that post-MI hearts of middle-aged rats demonstrated a noticeable reduction in numerical density of coronary capillaries mainly in the regions of the noninfarcted free wall in which the remaining cardiac myocytes underwent substantial concentric enlargement, i.e., in the epi- and endomyocardium of males and endomyocardium of female rats. However, in contrast to males, post-MI female rats had a smaller reduction in capillary density in free wall endomyocardium, despite the fact that both sexes had the noticeable concentric growth of cardiac myocytes in this region. This finding indicates a better regional adaptive expansion of capillaries in the subendocardium of female compared to male rats. Such observation was associated with a significant interaction between the effects of sex and the experimental model on the capillaryto-cardiac myocyte ratio in free wall endomyocardium.

In addition, we found that in both sexes the capillaryto-myocyte ratio increased dramatically only in the areas of free wall associated with enlargement of myocyte cross-sectional area greater than $50 \%$ of shame values. Considering the data reported previously on young adult male rats [39], it is feasible to suggest that such a significant increase in the capillary-to-myocyte ratio in these hypertrophic areas of post-MI male and female middle-aged rats, indicates augmented regional compensatory angiogenesis, 
which could be caused by a variety of factors, including increased wall stress and myocardial stretch, or impaired myocyte oxygenation [10]. Though, the effect of more prominent side-by-side myocyte slippage, which might substantially modify myocyte numerical density in these regions, cannot be ruled out either $[38,43]$.

While the capillary network is important for oxygen delivery to cardiac myocytes, the coronary arteriolar bed is critical for a distribution of blood between capillary domains. Post-MI hypertrophy of the LV myocardium requires structural modifications of the arteriolar tree in order to provide an adequate blood supply to the overloaded myocytes within noninfarcted LV regions $[8,9,48]$. Unfortunately, nearly all previous experimental studies that addressed arterioles in post-MI myocardium were done exclusively on male animals, including our own earlier studies on middle-aged rats $[6,21,22,26]$. However, according to our current findings and previous observations on mice of both sexes [55], female hearts have a significantly smaller arteriolar bed than do males. The evident sexspecific differences in the size of the arteriolar bed seen in our study are consistent with the data published by others, regardless of the fact that most of these studies have had the extent of the coronary arteriolar bed reported either for male $[56,57]$ or female $[50,58]$ animals only. The existence of sex/gender related differences in the size of coronary arterial beds has also been confirmed in human studies [59], revealing that women have smaller coronary artery size than men even after adjusting for either body size [60] or left ventricular mass [61].

Despite the fact that arteriolar length and volume densities were significantly greater in male than in female middle-aged rats as indicated by sham values, both sexes demonstrated a relatively similar degree of adaptive arteriolar growth in the LV free wall during post-MI remodeling. However, we found a significant interaction between the effects of sex and the experimental model on the arteriolar diameter and the frequency distribution of arterioles in 'LV free wall. Specifically, we established the fact that in females, the growth was limited to small diameter vessels only. It is important to note that previous studies done separately on male and female animals have also confirmed the fact that while in post-MI male rats the arterioles grew throughout the entire vascular tree of the remaining LV free wall myocardium [26], in females, the only small size arterioles showed a tendency to expansion [50]. In addition, we uncovered a significant interaction between the effects of sex and the experimental model on arteriolar density in the septum. This finding was associated with the lack of the noticeable compensatory growth of the arterioles in the septum of post-MI female rats as compared to male counterparts. The exact nature of these regional sex-related differences in the responses of male and female coronary arteriolar trees to post-MI remodeling remains to be clarified. We can only speculate that such discrepancy in the pattern of arteriolar bed reorganization within the septum as opposed to the LV free wall of the female post-MI heart, in comparison to males, might be caused by a variety of factors, including the regional difference in wall stress [41] or temporary composition of growth-stimulating milieu [6].

\section{Study limitations}

The current study examined the sex-related differences in hypertrophic response of cardiac myocytes and adaptive expansion of coronary microvessels only at one time point during MI-induced LV remodeling. Therefore, it is difficult to corroborate whether continuing ventricular remodeling would further modify a scale of adaptive responses. Furthermore, because only rats with a large transmural MI were used, the effect of the moderate or small size infarcts on a degree of adaptive processes remained unknown. In addition, it is important to emphasize that this experimental study on middle-aged rats was not intended to investigate the role of sex hormones during a post-MI remodeling process; instead, we attempted to evaluate the responses of different structural components in the remodeled male and female left ventricle under the natural circumstances that might occur in middleaged humans.

\section{Conclusions}

Taken together our data provided, the first evidence documenting the existence of sex-specific differences in regional adaptation of cardiac myocytes and coronary microvessels in middle-aged rats following 4 weeks of post-MI remodeling. We suggest that such regional peculiarities in structural adaptations within the left ventricle may to some extent influence the sex/gender-related differences in post-MI progression to heart failure.

\section{Competing interests}

The authors declare that they have no competing interests.

\section{Authors' contributions}

EID designed the study, performed microscopy and digital image collection, evaluated the data, and drafted the manuscript; LPC conducted all experiments and carried out the tissue collection; KO performed morphological assays, including immunostaining and quantitative image analysis; RJT provided financial support to the project, participated in discussion of the obtained findings, and critically reviewed the manuscript. All authors read and approved the final manuscript.

\section{Acknowledgements}

This study was supported by NIH Grant R01-HL62587 for RobertJ. Tomanek. The authors thank Ms. Alice O'Connor for help with histological techniques and Dr. Min-Kyung Jung for assistance with statistical analysis.

\section{Author details}

${ }^{1}$ Department of Biomedical Sciences, New York Institute of Technology College of Osteopathic Medicine, Rockefeller Building, Room 215E, Northern Boulevard, Old Westbury, NY 11568-8000, USA. ${ }^{2}$ Department of Anatomy and Cell Biology, University of lowa Carver College of Medicine, lowa City, IA 52242, USA. 
Received: 12 November 2013 Accepted: 16 December 2013 Published: 3 January 2014

\section{References}

1. Sutton MG, Sharpe N: Left ventricular remodeling after myocardial infarction: pathophysiology and therapy. Circulation 2000, 101:2981-2988.

2. Anversa P, Olivetti G, Capasso JM: Cellular basis of ventricular remodeling after myocardial infarction. Am J Cardiol 1991, 68:7D-16D.

3. Pfeffer MA, Braunwald E: Ventricular remodeling after myocardial infarction. Experimental observations and clinical implications. Circulation 1990, 81:1161-1172.

4. Sun Y, Zhang JQ, Zhang J, Lamparter S: Cardiac remodeling by fibrous tissue after infarction in rats. J Lab Clin Med 2000, 135:316-323.

5. Anversa P, Beghi C, Kikkawa Y, Olivetti G: Myocardial infarction in rats. Infarct size, myocyte hypertrophy, and capillary growth. Circ Res 1986, 58:26-37.

6. Dedkov El, Zheng W, Tomanek RJ: Compensatory growth of coronary arterioles in postinfarcted heart: regional differences in DNA synthesis and growth factor/receptor expression patterns. Am J Physiol Heart Circ Physiol 2006, 291:H1686-H1693.

7. Karam $\mathrm{R}$, Healy BP, Wicker P: Coronary reserve is depressed in postmyocardial infarction reactive cardiac hypertrophy. Circulation 1990, 81:238-246.

8. Kalkman EA, Bilgin YM, van Haren P, van Suylen RJ, Saxena PR, Schoemaker RG: Determinants of coronary reserve in rats subjected to coronary artery ligation or aortic banding. Cardiovasc Res 1996, 32:1088-1095.

9. Nelissen-Vrancken HJ, Debets JJ, Snoeckx LH, Daemen MJ, Smits JF: Time-related normalization of maximal coronary flow in isolated perfused hearts of rats with myocardial infarction. Circulation 1996, 93:349-355.

10. Tomanek RJ, Dedkov El: Angiogenesis and Arteriogenesis in Cardiac Hypertrophy. In Modern concepts in angiogenesis. Edited by Simons M, Rubanyi GM. London: Imperial College Press; 2007:253-280.

11. Ostadal B, Netuka I, Maly J, Besik J, Ostadalova I: Gender differences in cardiac ischemic injury and protection-experimental aspects. Exp Biol Med (Maywood) 2009, 234:1011-1019.

12. Litwin SE, Katz SE, Litwin CM, Morgan JP, Douglas PS: Gender differences in postinfarction left ventricular remodeling. Cardiology 1999, 91:173-183.

13. Cavasin MA, Tao Z, Menon S, Yang XP: Gender differences in cardiac function during early remodeling after acute myocardial infarction in mice. Life Sci 2004, 75:2181-2192

14. Jain M, Liao R, Podesser BK, Ngoy S, Apstein CS, Eberli FR: Influence of gender on the response to hemodynamic overload after myocardial infarction. Am J Physiol Heart Circ Physiol 2002, 283:H2544-H2550.

15. Luchner A, Brockel U, Muscholl M, Hense HW, Doring A, Riegger GA, Schunkert H: Gender-specific differences of cardiac remodeling in subjects with left ventricular dysfunction: a population-based study. Cardiovasc Res 2002, 53:720-727.

16. Crabbe DL, Dipla K, Ambati S, Zafeiridis A, Gaughan JP, Houser SR, Margulies $\mathrm{KB}$ : Gender differences in post-infarction hypertrophy in end-stage failing hearts. J Am Coll Cardiol 2003, 41:300-306.

17. Bairey Merz CN, Shaw LJ, Reis SE, Bittner V, Kelsey SF, Olson M, Johnson BD, Pepine CJ, Mankad S, Sharaf BL, Rogers WJ, Pohost GM, Lerman A, Quyyumi AA, Sopko G, WISE Investigators: Insights from the NHLBI-Sponsored Women's Ischemia Syndrome Evaluation (WISE) Study: Part II: gender differences in presentation, diagnosis, and outcome with regard to gender-based pathophysiology of atherosclerosis and macrovascular and microvascular coronary disease. J Am Coll Cardiol 2006, 47:S21-S29.

18. Vaccarino V, Badimon L, Corti R, de Wit C, Dorobantu M, Hall A, Koller A, Marzilli M, Pries A, Bugiardini R, Working Group on Coronary Pathophysiology and Microcirculation: Ischaemic heart disease in women: are there sex differences in pathophysiology and risk factors? Position paper from the working group on coronary pathophysiology and microcirculation of the European Society of Cardiology. Cardiovasc Res 2011, 90:9-17.

19. Bujak M, Kweon HJ, Chatila K, Li N, Taffet G, Frangogiannis NG: Aging-related defects are associated with adverse cardiac remodeling in a mouse model of reperfused myocardial infarction. J Am Coll Cardiol 2008, 51:1384-1392.

20. Raya TE, Gaballa M, Anderson P, Goldman S: Left ventricular function and remodeling after myocardial infarction in aging rats. Am J Physio/ 1997, 273: $\mathrm{H} 2652-\mathrm{H} 2658$.

21. Dedkov El, Zheng W, Christensen LP, Weiss RM, Mahlberg-Gaudin F, Tomanek RJ: Preservation of coronary reserve by ivabradine-induced reduction in heart rate in infarcted rats is associated with decrease in perivascular collagen. Am J Physiol Heart Circ Physiol 2007, 293:H590-H598.

22. Christensen LP, Zhang RL, Zheng W, Campanelli JJ, Dedkov El, Weiss RM, Tomanek RJ: Postmyocardial infarction remodeling and coronary reserve: effects of ivabradine and beta blockade therapy. Am J Physiol Heart Circ Physiol 2009, 297:H322-H330.

23. Rubin $B S$, Lee $C E$, King JC: A reduced proportion of luteinizing hormone (LH)releasing hormone neurons express Fos protein during the preovulatory or steroid-induced LH surge in middle-aged rats. Biol Reprod 1994, 51:1264-1272.

24. Nass TE, LaPolt PS, Judd HL, Lu JK: Alterations in ovarian steroid and gonadotrophin secretion preceding the cessation of regular oestrous cycles in ageing female rats. J Endocrinol 1984, 100:43-50.

25. Matthews KA, Wing RR, Kuller LH, Meilahn EN, Plantinga P: Influence of the perimenopause on cardiovascular risk factors and symptoms of middle-aged healthy women. Arch Intern Med 1994, 154:2349-2355.

26. Dedkov El, Christensen LP, Weiss RM, Tomanek RJ: Reduction of heart rate by chronic beta1-adrenoceptor blockade promotes growth of arterioles and preserves coronary perfusion reserve in postinfarcted heart. Am J Physiol Heart Circ Physiol 2005, 288:H2684-H2693.

27. Spadaro J, Fishbein MC, Hare C, Pfeffer MA, Maroko PR: Characterization of myocardial infarcts in the rat. Arch Pathol Lab Med 1980, 104:179-183.

28. Boyle MP, Weisman HF: Limitation of infarct expansion and ventricular remodeling by late reperfusion. Study of time course and mechanism in a rat model. Circulation 1993, 88:2872-2883.

29. Adair TH, Wells ML, Hang J, Montani JP: A stereological method for estimating length density of the arterial vascular system. Am J Physiol 1994, 266:H1434-H1438.

30. Opie LH, Commerford PJ, Gersh BJ, Pfeffer MA: Controversies in ventricular remodelling. Lancet 2006, 367:356-367.

31. Chen YF, Redetzke RA, Sivertson RM, Coburn TS, Cypher LR, Gerdes AM: Post-myocardial infarction left ventricular myocyte remodeling: are there gender differences in rats? Cardiovasc Pathol 2011, 20:e189-e195.

32. Bogatyryov Y, Tomanek RJ, Dedkov El: Structural composition of myocardial infarction scar in middle-aged male and female rats: does sex matter? J Histochem Cytochem 2013, 61:833-848.

33. Panagiotakos DB, Pitsavos C, Kourlaba G, Mantas $Y$, Zombolos S, Kogias $Y$, Antonoulas A, Stravopodis P, Stefanadis C: Sex-related characteristics in hospitalized patients with acute coronary syndromes-the Greek Study of Acute Coronary Syndromes (GREECS). Heart Vessels 2007, 22:9-15.

34. Oda E, Goto M, Matsushita H, Takarada K, Tomita M, Saito A, Fuse K, Fujita S, Ikeda Y, Kitazawa H, Takahashi M, Sato M, Okabe M, Aizawa Y: The association between obesity and acute myocardial infarction is age- and gender-dependent in a Japanese population. Heart Vessels 2013, 28:551-558.

35. Roberts CS, Maclean D, Braunwald E, Maroko PR, Kloner RA: Topographic changes in the left ventricle after experimentally induced myocardial infarction in the rat. Am J Cardiol 1983, 51:872-876.

36. Roberts CS, Maclean D, Maroko P, Kloner RA: Early and late remodeling of the left ventricle after acute myocardial infarction. Am J Cardiol 1984, 54:407-410

37. $X u$ Y, Arenas IA, Armstrong SJ, Davidge ST: Estrogen modulation of left ventricular remodeling in the aged heart. Cardiovasc Res 2003, 57:388-394.

38. Olivetti G, Capasso JM, Meggs LG, Sonnenblick EH, Anversa P: Cellular basis of chronic ventricular remodeling after myocardial infarction in rats. Circ Res 1991, 68:856-869.

39. Xie Z, Gao M, Batra S, Koyama T: The capillarity of left ventricular tissue of rats subjected to coronary artery occlusion. Cardiovasc Res 1997, 33:671-676.

40. Rohde LE, Aikawa M, Cheng GC, Sukhova G, Solomon SD, Libby P, Pfeffer J, Pfeffer MA, Lee RT: Echocardiography-derived left ventricular end-systolic regional wall stress and matrix remodeling after experimental myocardial infarction. J Am Coll Cardiol 1999, 33:835-842.

41. Gaballa MA, Goldman S: Ventricular remodeling in heart failure. J Card Fail 2002, 8:S476-S485

42. Weisman HF, Bush DE, Mannisi JA, Weisfeldt ML, Healy B: Cellular mechanisms of myocardial infarct expansion. Circulation 1988, 78:186-201.

43. Olivetti G, Capasso JM, Sonnenblick EH, Anversa P: Side-to-side slippage of myocytes participates in ventricular wall remodeling acutely after myocardial infarction in rats. Circ Res 1990, 67:23-34.

44. Cheng W, Kajstura J, Nitahara JA, Li B, Reiss K, Liu Y, Clark WA, Krajewski S, Reed JC, Olivetti G, Anversa P: Programmed myocyte cell death affects the viable myocardium after infarction in rats. Exp Cell Res 1996, 226:316-327. 
45. Olivetti G, Quaini F, Lagrasta C, Cigola E, Ricci R, Maestri R, Anversa P: Cellular basis of ventricular remodeling after myocardial infarction in rats. Cardioscience 1995, 6:101-106.

46. Tomanek RJ: Myocardial Ischemia and Infarction. In Coronary Vasculature: Development, Structure-Function, and Adaptations. New York: Springer; 2013:189-220

47. Anversa P, Capasso JM, Sonnenblick EH, Olivetti G: Mechanisms of myocyte and capillary growth in the infarcted heart. Eur Heart J 1990, 11(Suppl B):123-132.

48. Nelissen-Vrancken HJ, Kuizinga MC, Daemen MJ, Smits JF: Early captopril treatment inhibits DNA synthesis in endothelial cells and normalization of maximal coronary flow in infarcted rat hearts. Cardiovasc Res 1998, 40:156-164.

49. van Kerckhoven $R$, van Veghel R, Saxena PR, Schoemaker RG: Pharmacological therapy can increase capillary density in post-infarction remodeled rat hearts. Cardiovasc Res 2004, 61:620-629.

50. Chen YF, Weltman NY, Li X, Youmans S, Krause D, Gerdes AM: Improvement of left ventricular remodeling after myocardial infarction with eight weeks L-thyroxine treatment in rats. J Transl Med 2013, 11:40.

51. Rakusan K, Nagai J: Morphometry of arterioles and capillaries in hearts of senescent mice. Cardiovasc Res 1994, 28:969-972.

52. Tomanek RJ: Effects of age and exercise on the extent of the myocardial capillary bed. Anat Rec 1970, 167:55-62.

53. Anversa P, Li P, Sonnenblick EH, Olivetti G: Effects of aging on quantitative structural properties of coronary vasculature and microvasculature in rats. Am J Physiol 1994, 267:H1062-H1073.

54. Przyklenk K, Groom AC: Microvascular evidence for a transition zone around a chronic myocardial infarct in the rat. Can J Physiol Pharmacol 1983, 61:1516-1522.

55. Dedkov El, Thomas MT, Sonka M, Yang F, Chittenden TW, Rhodes JM, Simons M, Ritman EL, Tomanek RJ: Synectin/syndecan-4 regulate coronary arteriolar growth during development. Dev Dyn 2007, 236:2004-2010.

56. Larouche I, Schiffrin EL: Cardiac microvasculature in DOCA-salt hypertensive rats: effect of endothelin ET(A) receptor antagonism. Hypertension 1999, 34:795-801.

57. Liu Y, Wang D, Redetzke RA, Sherer BA, Gerdes AM: Thyroid hormone analog 3,5-diiodothyropropionic acid promotes healthy vasculature in the adult myocardium independent of thyroid effects on cardiac function. Am J Physiol Heart Circ Physiol 2009, 296:H1551-H1557.

58. Rakusan K, Wicker P: Morphometry of the small arteries and arterioles in the rat heart: effects of chronic hypertension and exercise. Cardiovasc Res 1990, 24:278-284.

59. Dickerson JA, Nagaraja HN, Raman SV: Gender-related differences in coronary artery dimensions: a volumetric analysis. Clin Cardiol 2010, 33:E44-E49.

60. Yang F, Minutello RM, Bhagan S, Sharma A, Wong SC: The impact of gender on vessel size in patients with angiographically normal coronary arteries. J Interv Cardiol 2006, 19:340-344.

61. Kucher N, Lipp E, Schwerzmann M, Zimmerli M, Allemann Y, Seiler C: Gender differences in coronary artery size per $100 \mathrm{~g}$ of left ventricular mass in a population without cardiac disease. Swiss Med Wkly 2001, 131:610-615.

doi:10.1186/2042-6410-5-1

Cite this article as: Dedkov et al:: Coronary vessels and cardiac myocytes of middle-aged rats demonstrate regional sex-specific adaptation in response to postmyocardial infarction remodeling. Biology of Sex Differences 2014 5:1.

\section{Submit your next manuscript to BioMed Central and take full advantage of:}

- Convenient online submission

- Thorough peer review

- No space constraints or color figure charges

- Immediate publication on acceptance

- Inclusion in PubMed, CAS, Scopus and Google Scholar

- Research which is freely available for redistribution 